\title{
CD147-cyclophilin A interactions promote proliferation of cutaneous T-cell lymphoma
}

\author{
Minami Sakamoto, Tomomitsu Miyagaki, Hiroaki Kamijo, Tomonori Oka, Naomi Takahashi, Hiraku Suga, Makoto \\ Sugaya, Shinichi Sato \\ Department of Dermatology, The University of Tokyo, Tokyo, Japan
}

\section{INTRODUCTION :}

- CD147 (a member of the immunoglobulin super family), presenting on the surface of many tumor cells, plays an important role in tumor progression through various functions, such as stimulating matrix metalloproteinases, inducing angiogenesis, and developing chemoresistance.

- Cyclophilin A (CypA), an intracellular protein promoting inflammation when released from cells, is known to be one of the natural ligands for CD147

- There have been no reports on CD147 and CypA involvement in cutaneous T-cell lymphoma (CTCL), such as mycosis fungoides (MF) and Sézary syndrome (SS).

\section{OBJECTIVE :}

To examine the expression and function of CD147 and CypA in CTCL.

\section{METHODS :}

- CD147 expression on CTCL cell lines (HH, Hut78, MJ cells) and CD4+CD7- tumor cells in peripheral blood from patients with SS $(n=4)$ was examined by flow cytometry.

- CypA production from CTCL cell lines was measured by ELISA.

- CD147 and CypA expression levels in lesional skin $(\mathrm{n}=22 ; 18 \mathrm{MF}$ cases and $4 \mathrm{SS}$ cases $)$ and sera $(\mathrm{n}=$ $53 ; 47 \mathrm{MF}$ cases and $6 \mathrm{SS}$ cases) were evaluated by real time RT-PCR, immunohistochemistry and/or ELISA.

- The involvement of CypA-CD147 interactions in the growth of CTCL cell lines was examined using anti-CD147 Ab and CypA inhibitor.

\section{RESULTS :}

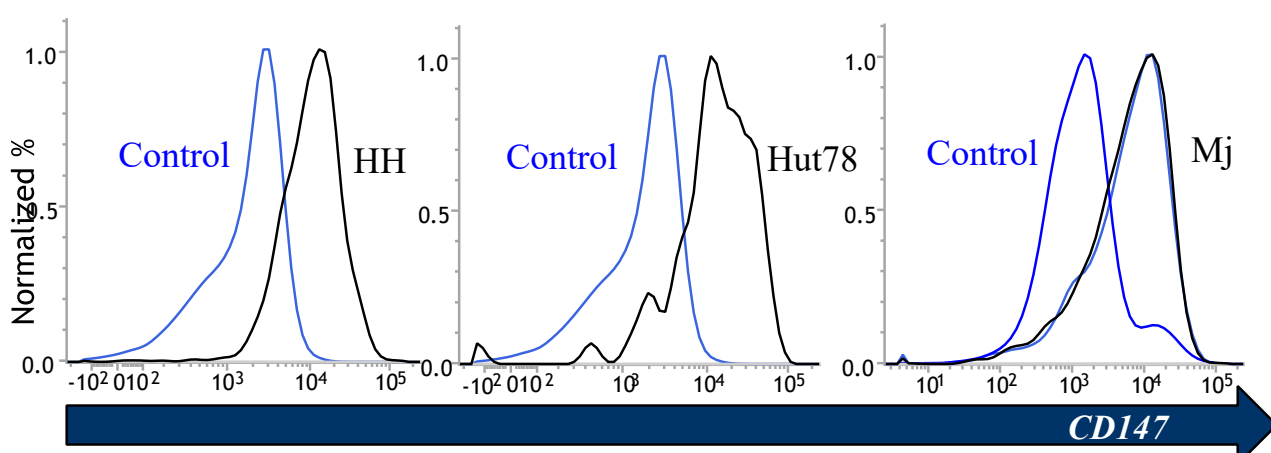

FIGURE 1. CD147 was expressed on CTCL cell lines. CD147 expression on CTCL cell lines were measured by flow cytometry.
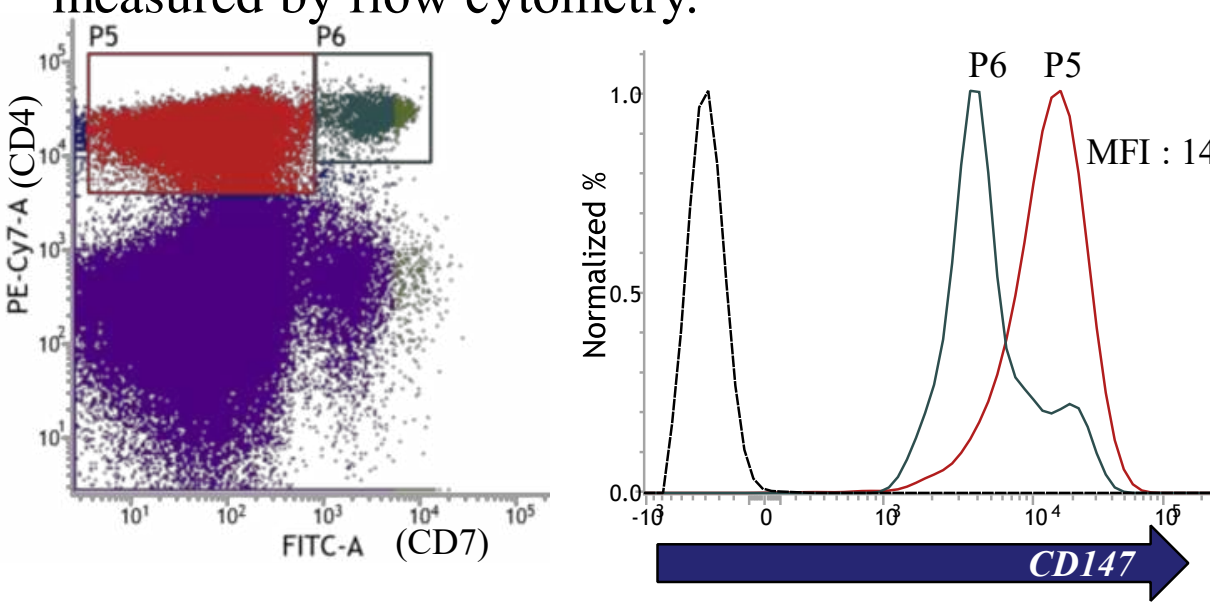

FIGURE 2. CD147 was expressed strongly on CD4+CD7- $T$ cells in peripheral blood from patients with Sézary syndrome.

CD147 expression on peripheral blood T cells from patients with Sézary syndrome was measured by flow cytometry. Representative data from four patients.

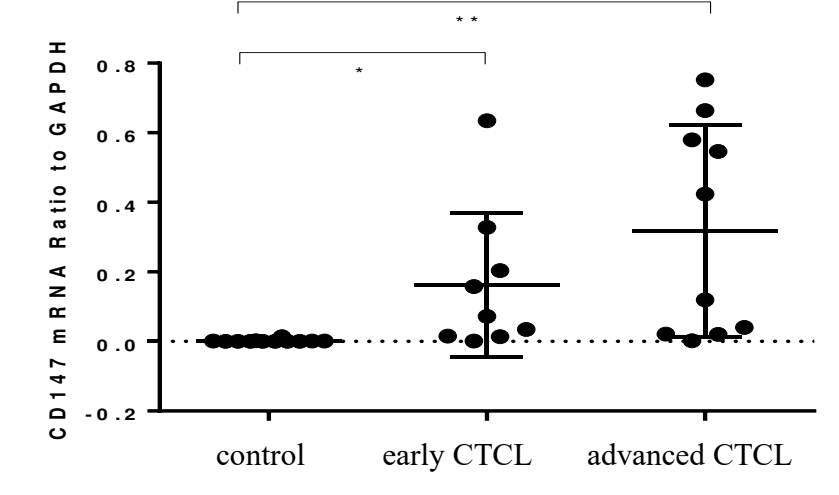

FIGURE 3. CD147 mRNA expression was increased in CTCL lesional skin. CD147 mRNA expression levels in the lesional skin of CTCL were measured by real-time RT-PCR.

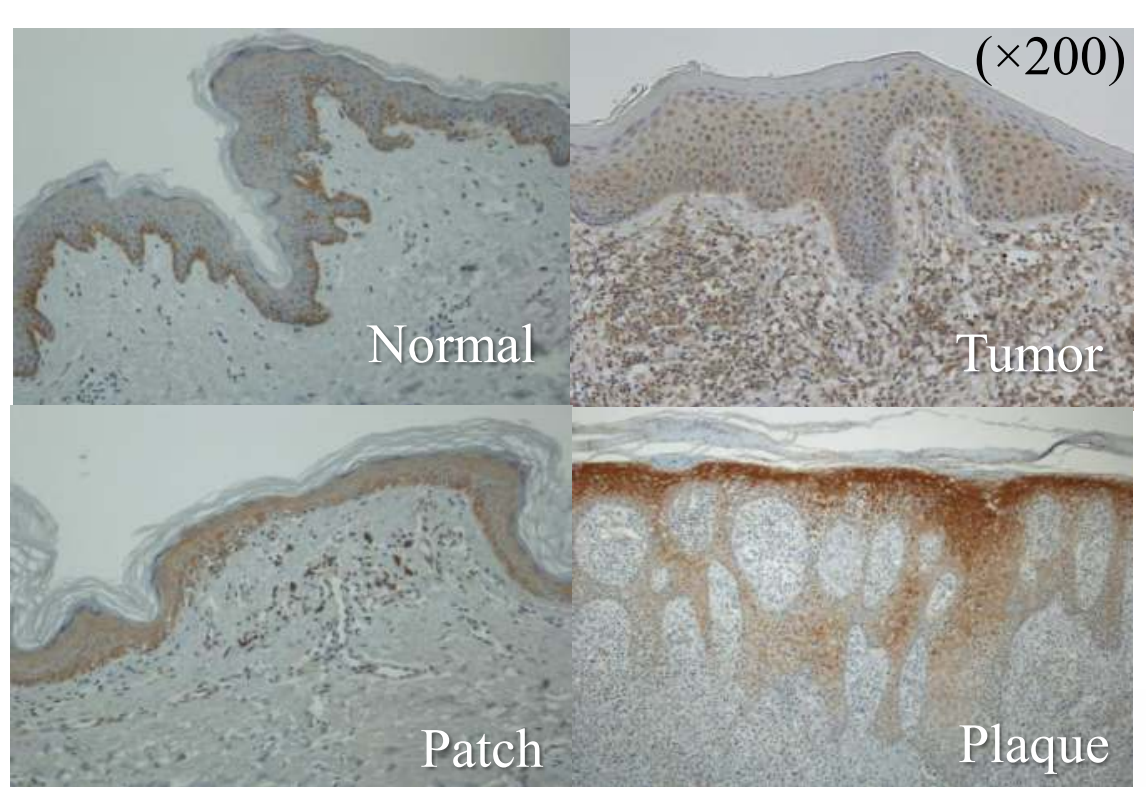

FIGURE 4. Immunohistochemistry revealed enhanced CypA expression in epidermal keratinocytes and tumor cells in lesional skin.

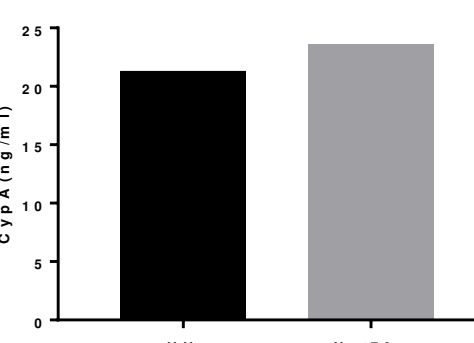

FIGURE 5. CTCL cell lines produced CypA. CypA levels in culture media of CTCL cell lines were measured by ELISA

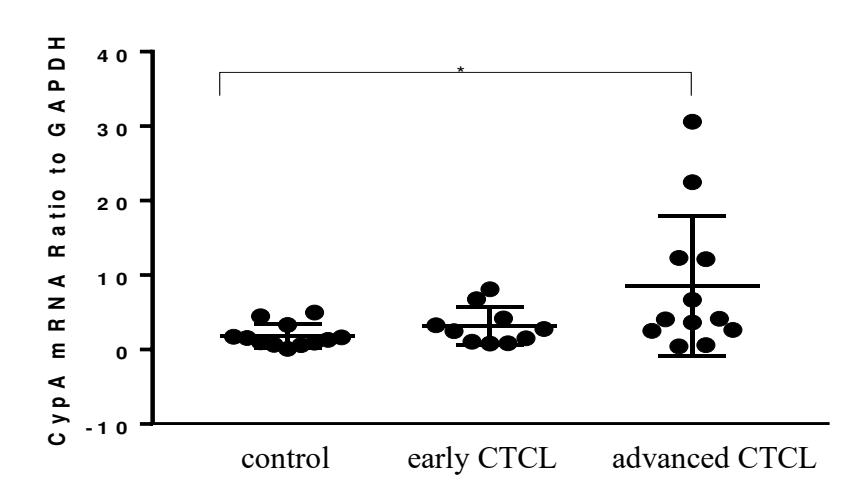

FIGURE 6. CypA mRNA expression was increased in CTCL lesional skin. CypA mRNA expression was measured by RT-PCR.
(A)

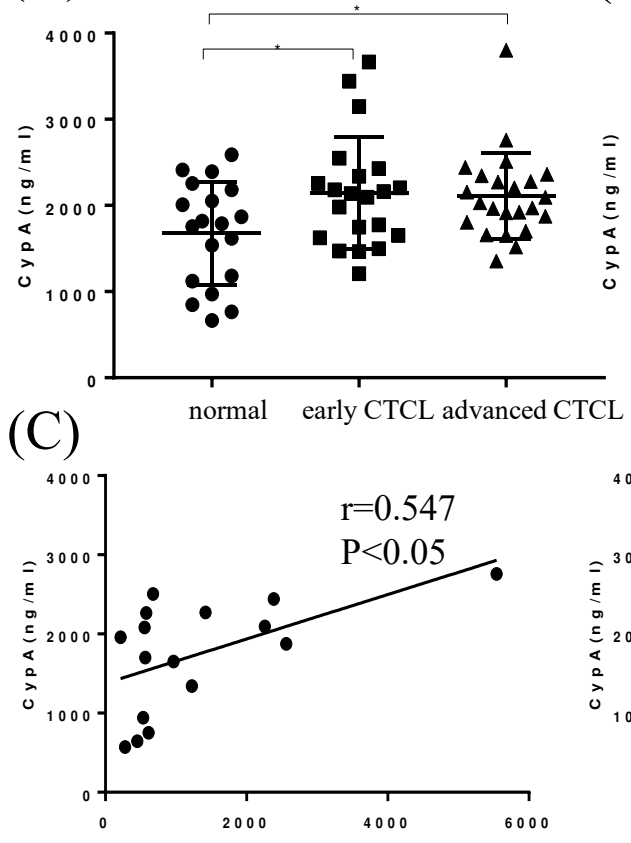

(B)

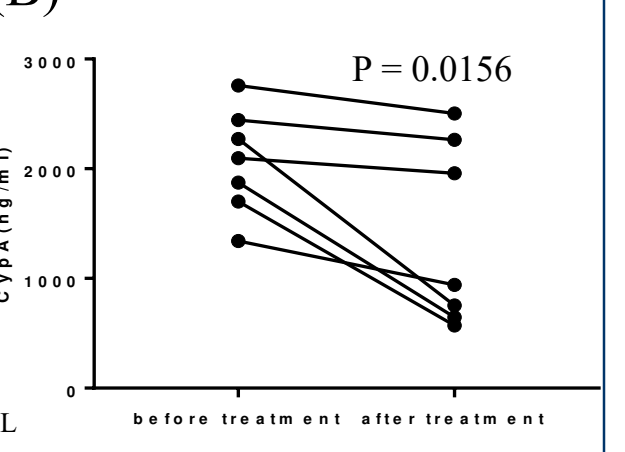

FIGURE 7. Serum CypA levels were increased in CTCL patients and correlated with disease severity. (A) Serum CypA levels in CTCL patients and healthy controls were measured by ELISA. (B) Serum CypA levels decreased after treatment. (C) Serum CypA levels significantly correlated with levels of serum LDH or sIL-2R in CTCL.

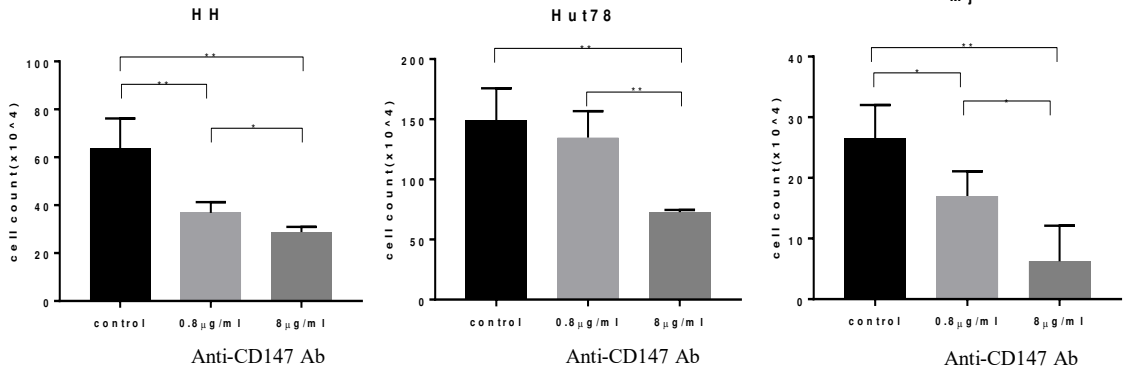

FIGURE 8. Anti-CD147 Ab suppressed cell growth of CTCL cell lines. CTCL cell lines were cultured with anti-CD147 Ab $(0.8$ or $8.0 \mu \mathrm{g} / \mathrm{ml})$ or medium. Forty-eight hours later, cell number was counted.

(A)

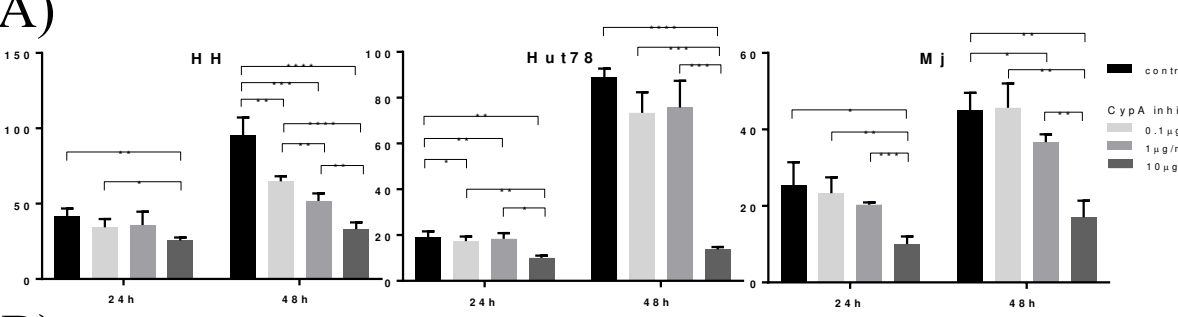

(B)

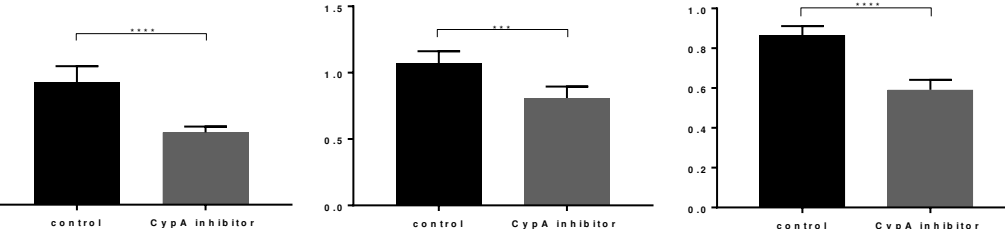

FIGURE 9. CypA inhibitor suppressed cell growth of CTCL cell lines. (A) CTCL cell lines were cultured with CypA inhibitor $(0.1,1.0$, or 10 $\mu \mathrm{g} / \mathrm{ml}$ ) or medium. Twenty-four or forty-eight hours later, cell number was counted. (B) BrdU cell proliferation assay of CTCL cell lines cultured with CypA inhibitor $(10 \mu \mathrm{g} / \mathrm{ml})$ or medium.

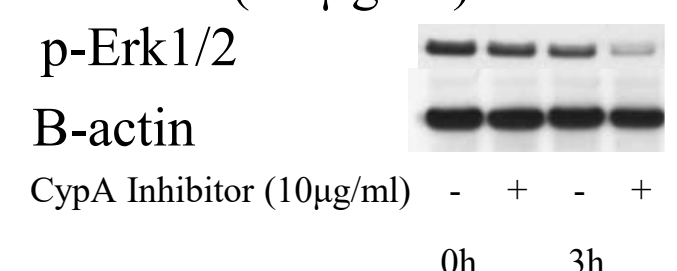

FIGURE 10. CypA inhibitor abrogated phosphorylation of ERK1/2 in HH cells. Abrogation of ERK1/2 phosphorylation in $\mathrm{HH}$ cells stimulated for 3 hours with $10 \mu \mathrm{g} / \mathrm{ml} \mathrm{CypA}$ inhibitor by Western blotting.

DISCUSSION :

\section{- Summary}

CTCL cell lines and tumor cells from patients with CTCL expressed both CD147 and CypA. CD147 and CypA mRNA levels were increased in lesional skin of CTCL. Serum CypA levels in CTCL patients were significantly higher than those of healthy controls and correlated with disease severity. CypA inhibitor or anti-CD147 Ab suppressed cell growth of CTCL cell lines. CypA inhibitor suppressed the phosphorylation of ERK $1 / 2$ in HH cells.

\section{-Conclusion}

This study suggests that interactions between CD147 expressed on the cell surface of CTCL tumor cells and CypA secreted by those cells may lead to the development of CTCL by supporting proliferation of tumor cells through upregulating ERK1/2 phosphorylation and that CD147-CypA interactions can be a therapeutic target for CTCL. 